Hospital in London. ${ }^{4}$ In only $3 \%$ of cases were paraffin sections necessary to establish the definitive diagnosis, and in no instance was cancer wrongly diagnosed. No patient was subjected to an unnecessary radical mastectomy on the basis of the pathologist's report. Desai finds that the classical freezing microtome is more useful than the newer cryostat technique in dealing with breast lesions. This is because the main source of error in the diagnosis of breast conditions is a failure to locate the lesion rather than a misinterpretation of the histological picture. For this reason an adequate number of sections of the lesion should be examined, an aim that is most easily achieved with the freezing microtome.

Thus the frozen-section technique, which yields correct results in experienced hands in $97-98 \%$ of cases, ${ }^{34}$ remains unchallenged in the rapid diagnosis of lesions before their treatment by surgical means. It is to be hoped that this service will be provided on an increasing scale so that it may be used more extensively than it is at present.

\section{Treatment of Empyema}

The incidence of pyogenic empyema has fallen sharply as the administration of antibiotics for respiratory infections has increased. The fall is also related to the prevention of the development of bronchiectasis in childhood and adolescence.

There are still some patients who develop a collection of pus in one or other pleural cavity in spite of early and adequate antibiotic therapy. But because antibiotics have been given the pus may be sterile in at least half of them. These patients do not usually show the florid signs of toxicity which were so evident in the preantibiotic era. Now there are two lines of treatment for empyema-firstly, drainage by rib resection, and, secondly, excision of the empyema, or decortication.

The first alternative is easily performed and effective, provided drainage is at the lowest dependent point of the empyema and the cavity is cleaned of all fibrinous masses before a large-bore drainage tube is inserted. In adult patients the operation is performed under local anaesthesia with the patient sitting up. There is still a limited place for it in the treatment of a toxic patient who is debilitated and acutely ill, either from neglect or from the presence of a resistant infection. Usually, however, the pus can be sterilized by aspiration together with instillation and injection of the appropriate antibiotics. By repeated thoracocentesis, daily if necessary, the tension in the abscess cavity can be reduced, and the danger of the empyema presenting through the chest wall or rupturing into the bronchial tree is minimized. By these means the acute stage of the illness can be converted to the subacute stage, when investigations leading up to complete excision of the empyema can be undertaken.

Primary infections of the pleura virtually never arise, so that a cause must be sought in the chest wall, mediastinum, and lung. It may be necessary to examine the adjacent ribs by tomography for evidence of osteomyelitis. A barium swallow will show oesophageal lesions. Most commonly the cause of the empyema is a preceding infection of the underlying lung, and a bronchogram after bronchoscopy must be carried out. Frequently the bronchogram will show only distortion and displacement of the bronchi, but occasionally unsuspected bronchiectasis may be seen and will require resection at the time of excision of the empyema. The fibrosis and contraction of the soft tissues of the thorax, which always accompany inflammation, can be reduced to the minimum by the prompt institution of postural and breathing exercises and by excision at an early date.

Excision of the empyema, or decortication, is performed as soon as the signs of general toxicity have subsided, and the earlier the operation is performed the easier it is to remove the walls of the abscess cavity. At thoracotomy through the bed of the appropriate rib (which is not resected, but merels reflected) the surgeon need not fear opening the empyema. for to do so usually makes the operation technically easier The lung in the vicinity of the empyema is freed, and the cortex overlying the visceral and parietal pleura is excised completely. Injury to the visceral pleura itself should be avoided, and the lung is allowed to expand to fill the empyema space rapidly and completely. The chest wall is closed with suction drainage through intercostal tubes. The operation of decortication is performed to restore function early, whereas tube drainage by rib resection may have to be performed as an emergency to save life.

A special problem is the ipsilateral empyema which develop: after pneumonectomy. If the primary cause is dehiscence of the bronchial suture line, urgent reamputation and resuture of the bronchial stump are indicated. For late cases, with or without bronchopleural fistula, it is probably safer to obliterate the empyema by drainage and thoracoplasty, but thoracoplasty is indicated only when the lung cannot be made to expand to the chest wall to obliterate the abscess cavity.

\section{Londonderry Meeting}

Next month an Annual Clinical Meeting of the British Medical Association is to be held for the first time in Northern Ireland. These short meetings were started some nine years ago to enable doctors to get together and talk about the problems of medical practice in all its varied aspects. Expert and novice alike have found they have something to learn from them as well as something to contribute Nor does learning go wholly unrequited, for some of the sessions are recognized by the Ministry of Health for the purposes of the Postgraduate Training Allowance for N.H.S. General Practitioners. A notice giving details appears in the programme, which is published at page 71 of the Supplement this week.

The Londonderry Division are to be the hosts of the meeting, which is being held in association with the Society of Occupational Medicine. Appropriately, therefore, the hazards of one of Northern Ireland's principal occupations, farming, are to be discussed at a plenary session. At another plenary session a group of medical and lay experts are to introduce a subject recently aired in the leader and correspondence columns of this journal-medicine and the offender against society. The subjects of head injury and chronic renal failure, with their perennial problems in both hospital and general practice, are among those of a more clinical character to be considered.

Surrounded by beautiful country, and offering a warmth of hospitality second to none, Londonderry is well worth a visit this spring. The meeting is from 13 to 16 April, and those wishing to attend should make their arrangements as soon as possible. Detailed information is included in the programme. 